06

\title{
Влияние толщины пленки Pt на изменение текстуры и доли кристаллической фазы при ее отжиге
}

\author{
(C) Р.В. Селюков, В.В. Наумов \\ Ярославский фрилиал Физико-технологического института РАН, \\ 150007 Ярославль, Россия \\ e-mail: rvselyukov@mail.ru
}

Поступило в Редакцию 24 апреля 2018 г.

В окончательной редакции 19 ноября 2019 г.

Принято к публикации 19 ноября 2019 г.

Текстурированные пленки Pt с толщиной 20-80 nm, нанесенные методом магнетронного распыления на окисленную пластину $c$ - $\mathrm{Si}(100)$, подвергались отжигу в вакууме в режиме $500^{\circ} \mathrm{C} / 60 \mathrm{~min}$. C помощью методов рентгеноструктурного анализа найдены зависимости параметров кристаллической текстуры и доли кристаллической фазы от толщины для исходных пленок и пленок, подвергнутых отжигу. Для нахождения доли кристаллической фазы в текстурированных пленках предложена оригинальная методика, основанная на анализе кривых качания. Найдено, что для всех толщин отжиг привел к улучшению текстуры и увеличению доли кристаллической фазы тем большим, чем меньше толщина. Данный результат объяснен появлением в результате отжига крупных вторичных зерен, чья объемная доля растет с уменьшением толщины. Для исходных пленок Pt исследована неоднородность распределений параметров текстуры и доли кристаллической фазы по глубине пленки.

Ключевые слова: платина, тонкие пленки, кристаллическая текстура, рентгеноструктурный анализ, кривые качания.

DOI: 10.21883/JTF.2020.05.49181.163-18

\section{Введение}

Тонкие поликристаллические пленки Рt применяются в качестве тыльного электрода в приборах с перовскитоподобными сегнетоэлектриками [1-4], $\mathrm{ZnO}$ [5-7] и AlN [8]. Как правило, Pt-электроды наносят на окисленный $c$-Si или на стекла с использованием адгезионных подслоев, например, Ті или $\mathrm{TiO}_{2}$. Обычно Pt в таких системах имеет аксиальную кристаллическую текстуру (111), и степень преимущественной ориентации зерен (часто называемая как острота текстуры) Pt оказывает влияние на структуру, а следовательно, на свойства осажденных на нее материалов. Пленки вышеупомянутых материалов, как правило, осаждают на Pt-электрод при повышенной температуре, или подвергают отжигу после осаждения, приборы на основе таких структур часто работают при температурах до $1000^{\circ} \mathrm{C}$ [8]. Известно, что если температура превышает определенное значение, то в поликристаллических пленках и объемных материалах происходят рекристаллизация и последующий рост зерен. В процессе роста зерен наряду с так называемым нормальным ростом может наблюдаться аномальный рост, приводящий к формированию сравнительно крупных зерен, которые принято называть вторичными [9]. Основная фракция зерен при этом имеет меньшие размеры и формируется в результате нормального роста, такие зерна принято называть обычными. Нормальный рост зерен обусловлен уменьшением энергии межзеренных границ, аномальный рост зерен в тонких пленках обусловлен, главным образом, уменьшением поверхностной энергии, что имеет два важных следствия. Во-первых, для вторичных зерен, как правило, характерна ориентация параллельно подложке тех атомных плоскостей, которые обеспечивают минимальную поверхностную энергию, например, для ГЦК металлов это плоскости (111). Во-вторых, теория аномального роста, учитывающая влияние поверхностной энергии, предсказывает увеличение скорости роста латеральных размеров вторичных зерен с уменьшением толщины пленки [9]. Таким образом, одинаковая термообработка пленок разной толщины приводит к существенно разным изменениям их микроструктуры и текстуры. Это необходимо учитывать при изготовлении и эксплуатации упомянутых выше приборов, однако систематических исследований таких эффектов в пленках Pt авторами не найдено.

Рост зерен при термообработке приводит к уменьшению площади, а следовательно, и объема межзеренных границ. В результате уменьшается количество материала, находящегося в неупорядоченном состоянии в межзеренных границах [10], следовательно, увеличивается доля материала $\delta$, находящегося в кристаллической фазе. Знание параметра $\delta$ важно во многих случаях из-за его влияния на физические свойства пленок [11], а также при изучении процессов их кристаллизации $[12,13]$. При этом измерение $\delta$ в пленках затруднительно по нескольким причинам. Для нанокристаллических материалов существуют методики определения $\delta$, основанные на измерении размеров зерен $[14,15]$, а также на измерении модуля Юнга [16]. Область применения этих методик 
ограничена материалами с равноосными зернами, тогда как пленки часто содержат большие фракции столбчатых зерен. В рентгеновской дифрактометрии для образцов, не имеющих текстуры, $\delta$ определяется путем сравнения интегральных интенсивностей пиков на $\theta-2 \theta$ дифрактограммах для исследуемого образца и эталона из того же материала с известным значением $\delta$ [17]. При отсутствии эталона остается возможность определить таким методом значения $\delta$ относительно одного из образцов, для которого $\delta$ принимается равным 100\%. Такая методика часто применяется для вычисления $\delta$ в пленках, имеющих один и тот же тип текстуры [11-13], однако при этом пренебрегается различием в качестве текстур разных образцов. Это различие учитывается в предлагаемой нами в настоящей работе рентгеновской методике определения относительных значений $\delta$ для текстурированных пленок с помощью кривых качания.

Первой целью настоящей работы было установить, как в зависимости от толщины пленки $\mathrm{Pt}$ меняется воздействие отжига в вакууме в режиме $500^{\circ} \mathrm{C} / 60 \mathrm{~min}$ на ее текстуру и $\delta$. Для этого до и после отжига с помощью дифрактограмм, полюсных фигур и кривых качания были определены угол рассеяния текстуры $\Delta \gamma[18]$ и $\delta$ для пленок Pt с толщиной $h=20-80 \mathrm{~nm}$. Второй целью работы было найти распределение параметров $\Delta \gamma$ и $\delta$ по глубине $t$ исходной пленки. Для этого была разработана методика вычисления $\Delta \gamma(t)$ и $\delta(t)$, основанная на предположении о неподвижности межзеренных границ внутри пленки Pt во время ее нанесения.

\section{1. Эксперимент}

Пленки $\mathrm{Pt}$ толщинами 20, 40, 60, $80 \mathrm{~nm}$ осаждались методом магнетронного распыления на окисленные пластины Si (100). Нанесение пленки проводилось в установке SCR 651 „Tetra“ (Alcatel) при комнатной температуре, остаточном давлении $5 \cdot 10^{-5} \mathrm{~Pa}$ и давлении аргона $0.2 \mathrm{~Pa}$. Скорость осаждения Pt составляла $0.5 \mathrm{~nm} / \mathrm{s}$. После нанесения пленки отжигались в вакууме при $500^{\circ} \mathrm{C}$ в течение $60 \mathrm{~min}$. Данная температура примерно соответствует порогу рекристаллизации в объемной Pt [19].

Толщина пленок измерялась по сколу с помощью сканирующего электронного микроскопа (СЭМ) Supra40 (Carl Zeiss). Рентгеноструктурные исследования пленок проводились до и после отжига. Съемки $\theta-2 \theta$ дифрактограмм, полюсных фигур [18] Pt (111) и $\mathrm{Pt}(200)$, a также кривых качания пиков $\mathrm{Pt}(111)$ проводились с помощью дифрактометра ДРОН-3М с фокусировкой по Брэггу-Брентано, оснащенного гониометрической приставкой ГП-14. Использовалась трубка с медным анодом, линия $\mathrm{Cu} K_{\beta}$ устранялась с помощью кристалламонохроматора. Падающее на образец излучение представляло собой сумму линий $\mathrm{Cu} K_{\alpha 1}+\mathrm{Cu} K_{\alpha_{2}}$, дублетность излучения в расчетах не учитывалась. При съемке полюсных фигур шаг сканирования по углу наклона $\alpha$ был равен $2^{\circ}$, шаг сканирования по углу вращения $\beta$ был равен $2.4^{\circ}$. Кривые качания были сняты с шагом угла поворота образца $\gamma$, равным $0.05^{\circ}$. С помощью полюсных фигур было найдено, что во всех случаях пленки обладают аксиальной (111) текстурой, также был определен угол наклона оси текстуры. С помощью кривых качания $\operatorname{Pt}(111)$ вычислялись $\Delta \gamma$ и $\delta$. Угол рассеяния текстуры определялся как полуширина соответствующей кривой качания после ее коррекции с учетом фона, поглощения излучения в пленке и изменения облучаемого объема $\mathrm{Pt}$, обусловленного поворотом образца во время съемки. При вычислении $\delta$ предполагалось, что данный параметр прямо пропорционален площади под скорректированной кривой качания. Использование кривой качания для вычисления $\delta$ позволяет учесть кристаллическую фазу в зернах всех имеющихся ориентировок плоскостей (111).

В пленках распределение $\Delta \gamma$ и $\delta$ по $t$ неоднородно, поэтому, строго говоря, пленку необходимо характеризовать зависимостями $\Delta \gamma(t)$ и $\delta(t)$. Получить такие распределения без дополнительных предположений затруднительно, поэтому при расчетах пренебрегали зависимостью $\Delta \gamma$ и $\delta$ от $t$ и вычисляли эффективные значения $\Delta \gamma_{\text {eff }}$ и $\delta_{\text {eff. }}$ Однако в случае исходных пленок можно вычислить и $\Delta \gamma(t)$ и $\delta(t)$, предполагая, что межзеренные границы во время нанесения пленки были неподвижны. В этом случае зависимости $\Delta \gamma(t)$ и $\delta(t)$ определялись в приближении ступенчатого распределения $\Delta \gamma$ и $\delta$ по глубине пленки с шириной ступени $20 \mathrm{~nm}$.

\section{2. Результаты и обсуждение}

На рис. 1 показаны центральные части полюсных фигур (ПФ) $\operatorname{Pt}(111)$ и $\operatorname{Pt}(200)$ для исходной и подвергнутой отжигу пленки толщиной $80 \mathrm{~nm}$. Видно, что исходная пленка имеет аксиальную (111) текстуру, причем ось текстуры отклонена от нормали к поверхности на $1-2^{\circ}$. Отжиг не меняет тип и угол наклона оси текстуры, увеличивается только острота текстуры (рис. $1, a, c)$, что проявляется в сужении и увеличении текстурного максимума на ПФ $\mathrm{Pt}(111)$. Эти утверждения справедливы и для пленок других толщин.

\section{1. Влияние толщины пленки $\mathrm{Pt}$ на изменение качества текстуры и относительной доли кристаллической фазы в результате отжига}

Так как для всех образцов наблюдается наклон оси текстуры от нормали к поверхности, для правильного определения $\Delta \gamma$ съемка кривой качания всегда проводилась при значении угла вращения $\beta$ образца, для которого ось текстуры все время съемки находится в плоскости дифракции. При этом кривая качания представляет собой сечение центральной части ПФ $\operatorname{Pt}(111)$, проходящее через начало координат и текстурный максимум. Следуя подходу, предложенному в работе [20] для анализа полюсных фигур, найдем выражение для 


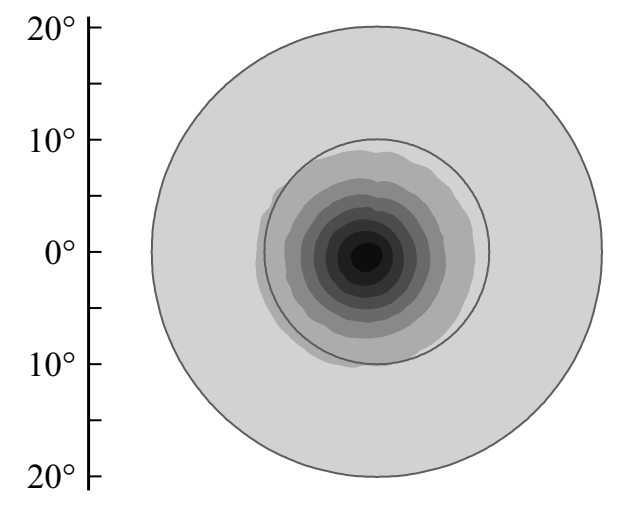

$c$

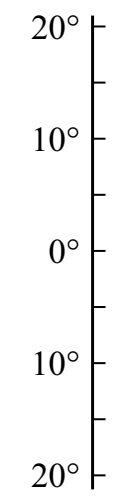

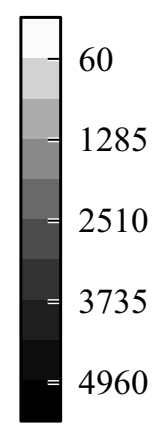

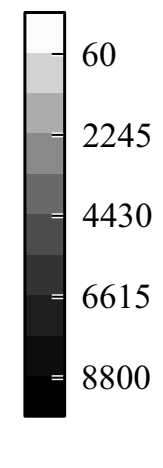

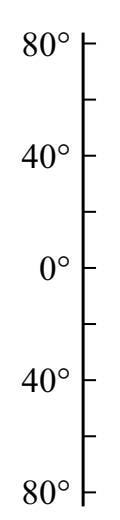

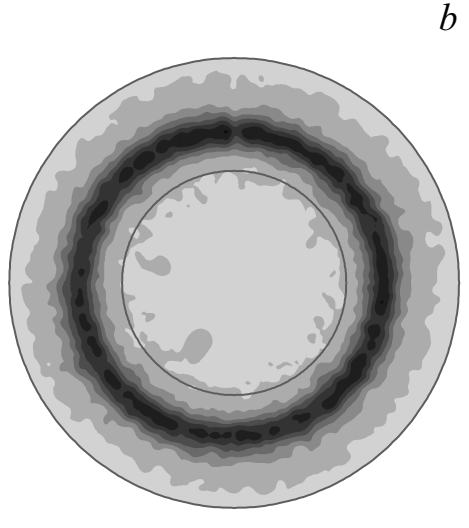

$b$

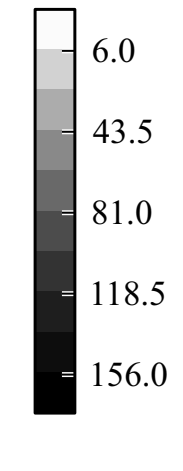

$d$

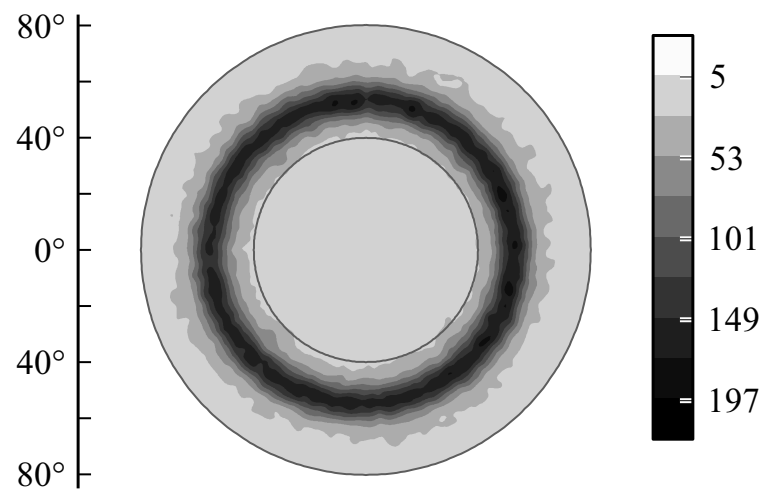

Pис. 1. Центральные части полюсных фигур $\mathrm{Pt}(111)(a, c)$ и $\mathrm{Pt}(200)(b, d)$ для пленки толщиной $80 \mathrm{~nm}$, исходной $(a, b)$ и подвергнутой отжигу $500^{\circ} \mathrm{C} / 60 \mathrm{~min}(c, d)$.

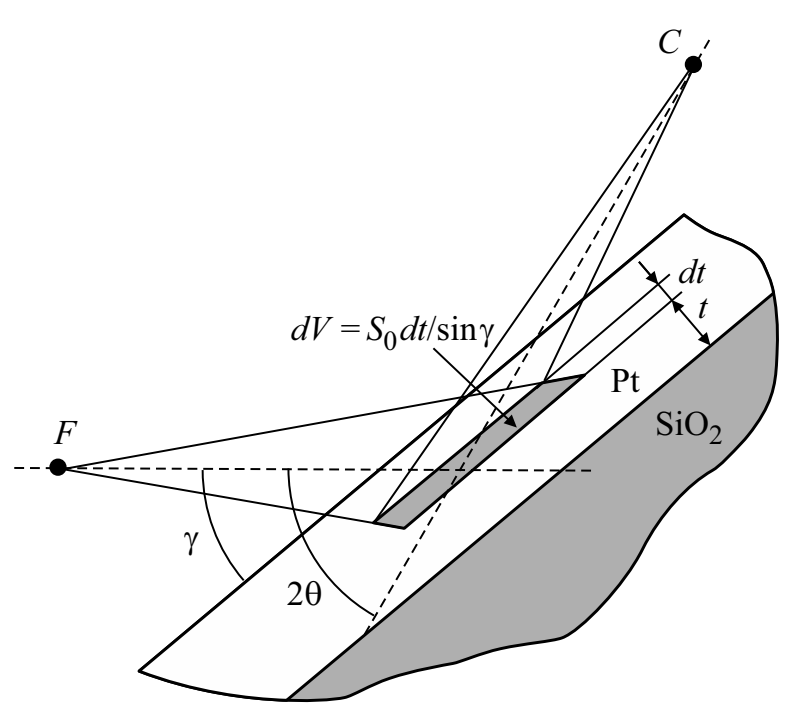

Рис. 2. Схема съемки кривой качания образца. $F-$ фокус рентгеновской трубки, $C$ - счетчик импульсов. $S_{0}, t, d t, d V, \theta$, $\gamma$ (см. пояснения в тексте).

кривой качания $R C$, в котором учтены поглощение излучения в пленке, а также изменение облучаемого объема Pt, обусловленное поворотом образца в процессе съемки. Рассмотрим элемент объема пленки $d V$ (рис. 2), на который падает расходящийся пучок излучения интенсивностью $I$ :

$$
I=I_{0} \exp [-(h-t) \mu / \sin \gamma]
$$

где $I_{0}-$ интенсивность падающего на поверхность образца излучения, $h-$ толщина пленки, $\mu-$ коэффициент поглощения рентгеновского излучения для $\mathrm{Pt}$, $\mu=4290 \mathrm{~cm}^{-1}[21], \gamma$ - угол поворота образца при съемке кривой качания, $\gamma$ изменяется в пределах от 0 до 20. В направлении счетчика импульсов распространяется дифрагированное излучение интенсивностью $d I^{\prime}$ :

$$
\begin{aligned}
d I^{\prime}= & I_{0} D(t, \gamma) \exp [-(h-t) / L(\gamma)] d V=\frac{I_{0} S_{0} D(t, \gamma)}{\sin \gamma} \\
& \times \exp [-(h-t) / L(\gamma)] d t
\end{aligned}
$$

где $1 / L(\gamma)=\mu / \sin (\gamma)+\mu / \sin (2 \theta-\gamma), S_{0}$ - площадь поперечного сечения пучка перед образцом, $d t$ - элемент толщины пленки, $D$ - отношение интенсивности дифрагированного в $d V$ излучения к интенсивности излучения, падающего на $d V . D$ прямо пропорционально количеству кристаллической фазы в $d V$, содержащемуся в кристаллитах, плоскости (111) которых отклонены от плоскости пленки на угол $|\theta-\gamma|[18,20]$. Интегрирование 
по всему облучаемому объему пленки дает выражение для $R C$ :

$$
\begin{aligned}
R C(h, \gamma)-R C_{b g}(h, \gamma)= & \frac{I_{0} S_{0}}{\sin \gamma} \int_{0}^{h} D(t, \gamma) \\
& \times \exp [-(h-t) / L(\gamma)] d t,
\end{aligned}
$$

где $R C_{b g}(h, \gamma)$ - фон, измеренный как кривая качания при положении счетчика под углом $2 \theta=2 \theta_{111}+3^{\circ}$. В формуле (3) интегрирование по $d t$ проводится от границы раздела пленки и подложки до поверхности пленки. Функция $D(t, \gamma)$ представляет собой распределение отклонений нормалей атомных плоскостей (111) от оси текстуры в слое $d t$ на расстоянии от поверхности пленки $h-t$. В общем случае $D$ зависит от $t$, однако без постановки специальных экспериментов невозможно определить эту зависимость, поэтому, как правило, предполагается, что $D$ не зависит от $t$, и $D(t, \gamma)$ заменяется эффективным значением $D_{\mathrm{eff}}(\gamma)$ :

$$
D_{\mathrm{eff}}(h, \gamma)=\frac{\sin \gamma}{I_{0} S_{0} L} \frac{R C(h, \gamma)-R C_{b g}(h, \gamma)}{1-\exp (-h / L)}
$$

$D_{\text {eff }}(\gamma)$ представляет собой кривую качания, исправленную с учетом фона, поглощения в пленке и изменения облучаемого объема. Зависимости $D_{\mathrm{eff}}(\gamma)$ для исходных пленок приведены на рис. 3, $a$. Углы рассеяния текстуры $\Delta \gamma_{\text {eff }}$ вычислялись как полуширины соответствующих профилей $D_{\text {eff }}(\gamma)$, результаты вычисления $\Delta \gamma_{\mathrm{eff}}(h)$ для исходных и подвергнутых отжигу пленок приведены на рис. $3, b$. Зависимость $\Delta \gamma_{\mathrm{eff}}(h)$ для исходной $\mathrm{Pt}$ является убывающей, что типично для поликристаллических пленок на неориентирующих подложках: такие же результаты были получены, например, для пленки Ti, нанесенной на $\mathrm{SiO}_{2}$ [22] и $\mathrm{AlN}$ на Pt [23]. Улучшение качества текстуры с ростом $h$ может быть объяснено следующим образом. На начальной стадии роста на неориентирующей подложке формируются островки с разными кристаллографическими ориентациями, однако при данных условиях нанесения пленки энергетически выгоден рост кристаллитов с наименьшей поверхностной энергией [24]. Для ГЦК металлов это будут кристаллиты с атомными плоскостями (111), параллельными поверхности подложки. По этой причине в процессе роста островков доля материала в зернах с такой ориентацией увеличивается, таким образом, пленка приобретает преимущественную ориентацию. Дальнейший рост происходит на сформировавшихся зернах и является ориентированным. В результате происходит улучшение качества текстуры пленки с увеличением ее толщины [24,25].

На рис. 3, с видно, что для всех $h$ отжиг привел к улучшению качества текстуры, причем степень этого улучшения возрастает с уменьшением $h$. В [26] было установлено, что в процессе отжига в исследуемой пленке Pt происходит рост обычных и вторичных зерен, причем латеральные размеры и суммарная площадь вторичных зерен тем больше, чем меньше $h$. В работе [9] была предложена модель, согласно которой вторичные зерна обладают наименьшей поверхностной энергией, и скорость их роста будет тем выше, чем меньше толщина пленки. Для зерен с другими ориентациями модель не предусматривает такого эффекта. Очевидным следствием из этой модели будет то, что текстура пленки должна при отжиге улучшаться тем сильнее, чем меньше ее толщина, что и находит экспериментальное подтверждение в нашей работе.

Как указывалось во Введении, применение стандартных рентгеновских методик определения $\delta$ в текстурированных материалах дает неверный результат из-за неучета зависимости $D_{\text {eff }}$ от угла $\gamma$. В настоящей работе предлагается методика оценки $\delta$ с помощью кривых качания, позволяющая учесть такую зависимость. Из-за отсутствия эталона с известным значением $\delta$ в рамках предлагаемой методики есть возможность оценить только относительную долю кристаллической фазы, когда для образца с наибольшим значением $\delta$ принимается, что $\delta=100 \%$. Чтобы вычислить $\delta$, необходимо провести суммирование интегральных интенсивностей от всех существующих в пленке ориентировок плоскостей $(h k l)$. Для пленки Pt с сильной аксиальной текстурой (111) это можно сделать с помощью ПФ (111). С учетом необходимых поправок доля кристаллической фазы будет прямо пропорциональна сумме $\sum_{\alpha, \beta} b(h, \alpha) D_{\text {eff }}(h, \alpha, \beta)$, где суммирование по $\alpha$ ведется так, чтобы захватить область текстурного максимума в центре ПФ (рис. $1, a, c)$, суммирование по $\beta$ ведется в диапазоне $0-360^{\circ}, b(h, \alpha)$ - интегральная ширина пика Pt (111) на $\theta-2 \theta$ дифрактограмме, снятой при наклоне образца с пленкой толщиной $h$ на угол $\alpha$. Как указывалось выше, кривая качания Pt (111) представляет собой сечение центральной части полюсной фигуры Pt (111), которое в нашем эксперименте проведено через центр ПФ и текстурный максимум. В случае аксиальной симметрии ПФ суммирование $\sum_{\alpha, \beta} b(h, \alpha) D_{\mathrm{eff}}(h, \alpha, \beta)$ сводится к суммированию по $\alpha$, а следовательно, будет эквивалентно интегрированию функции $b(h, \gamma) D_{\mathrm{eff}}(h, \gamma)$ по $\gamma$, поэтому c точностью до постоянного множителя, пренебрегая зависимостью $b$ от $\gamma$, можно записать

$$
\delta_{\mathrm{eff}}(h)=b(h) \int_{0}^{2 \theta} D_{\mathrm{eff}}(h, \gamma) d \gamma
$$

В формуле (5) $b(h)$ определяется с помощью дифрактограммы, снятой при $\gamma=\theta$ с использованием тех же щелей источника и счетчика, что использовались для съемки кривой качания. Так как $\gamma$ при съемке кривой качания не может выходить за пределы диапазона $0-2 \theta_{111}$, интегрирование в (5) захватывает ПФ только в диапазоне углов наклона $\alpha=0-\theta_{111}$. Таким образом, 

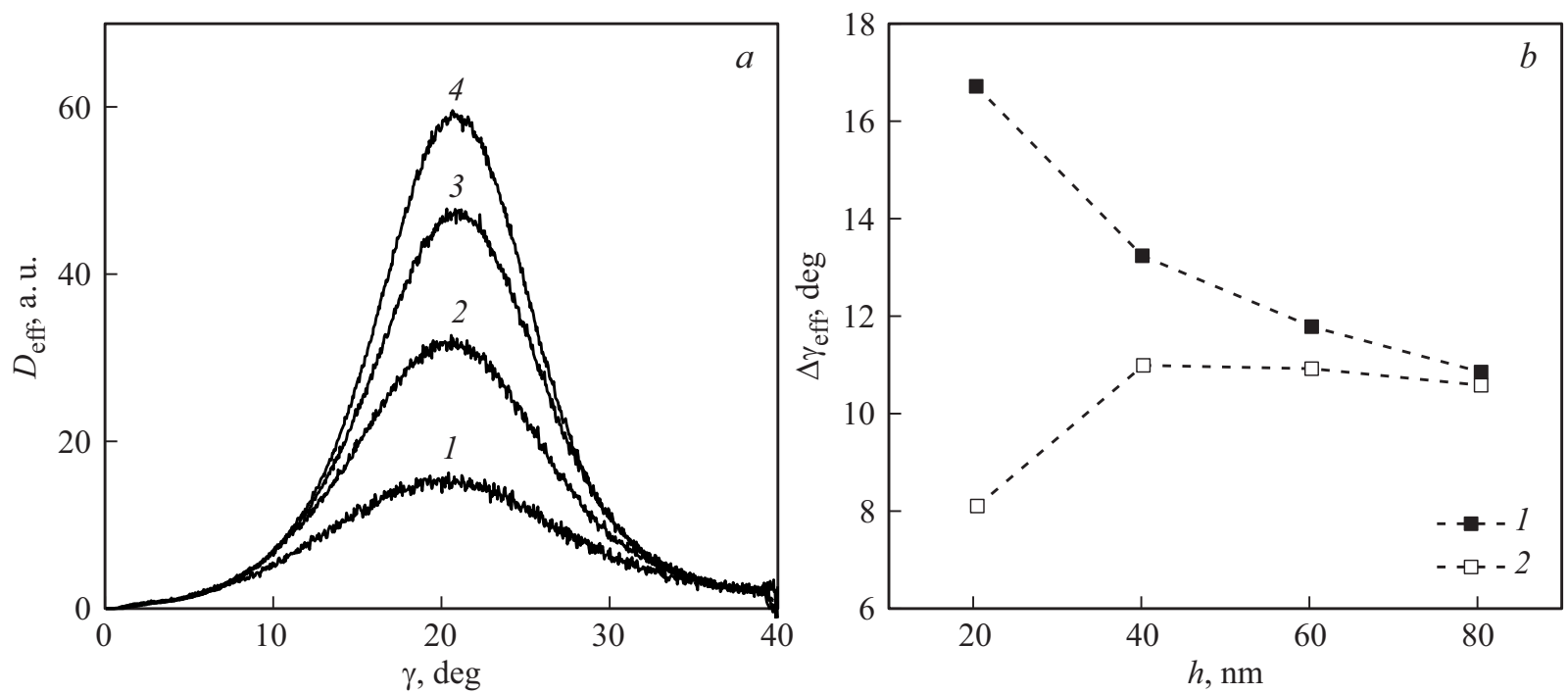

Рис. 3. $a-$ исправленные кривые качания $D_{\mathrm{eff}}(\gamma)$ для исходных пленок Рt толщинами 20 (1), 40 (2), 60 (3), $80 \mathrm{~nm}(4)$. $b-$ зависимости угла рассеяния текстуры $\Delta \gamma_{\text {eff }}$ от толщины пленки $h$ для исходной $(1)$ и подвергнутой отжигу $500^{\circ} \mathrm{C} / 60$ min $(2)$ пленки Pt.

данная методика применима только для пленок с однокомпонентной аксиальной текстурой, при этом хотя бы один текстурный максимум должен находиться в данном диапазоне углов $\alpha$. Так как для Pt $2 \theta_{111} \approx 40^{\circ}$, для полученных в нашем эксперименте ПФ Pt (111) это требование выполняется для всех образцов (рис. 1,a,c). Необходимо заметить, что в случае пленки $\mathrm{Pt}$ с аксиальной текстурой (111) происходит суммирование по тем зернам, чьи плоскости (111) отклонены от плоскости подложки на углы $0-20^{\circ}$ и $50-90^{\circ}$. Второй диапазон углов обусловлен учетом дифракции на тех плоскостях из совокупности $\{111\}$, которые дают на ПФ (111) текстурный максимум в виде кольца в области $\alpha \approx 70.5^{\circ}$ (на рис. $1, a, c$ не показан). Количество материала в неучтенных зернах представляется пренебрежимо малым, так как интенсивность на ПФ в диапазоне $\alpha=20-50^{\circ}$ не превышает фона.

Значения $\delta_{\text {eff }}$ были вычислены как по описанной выше методике, так и путем измерения интегральной

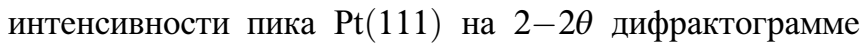
(см. Введение). Результаты для исходной и подвергнутой отжигу пленки Pt представлены на рис. 4. Видно, что значение $\delta_{\text {eff }}(h)$, определенное с помощью традиционной методики для $20 \mathrm{~nm}$ пленки, подвергнутой отжигу, существенно завышено, что объясняется наилучшим среди всех образцов качеством текстуры этой пленки. В случае лучшей текстуры при стандартной геометрии съемки $\theta-2 \theta$ дифрактораммы условию Брэгга удовлетворяет большая доля объема пленки, чем в случае худшей текстуры, следовательно, интегральная интенсивность пика на дифрактограмме будет выше, что и приведет к завышению значения $\delta_{\text {eff. }}$ В случае исходной пленки

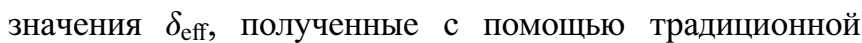
методики, несколько занижены, это можно объяснить

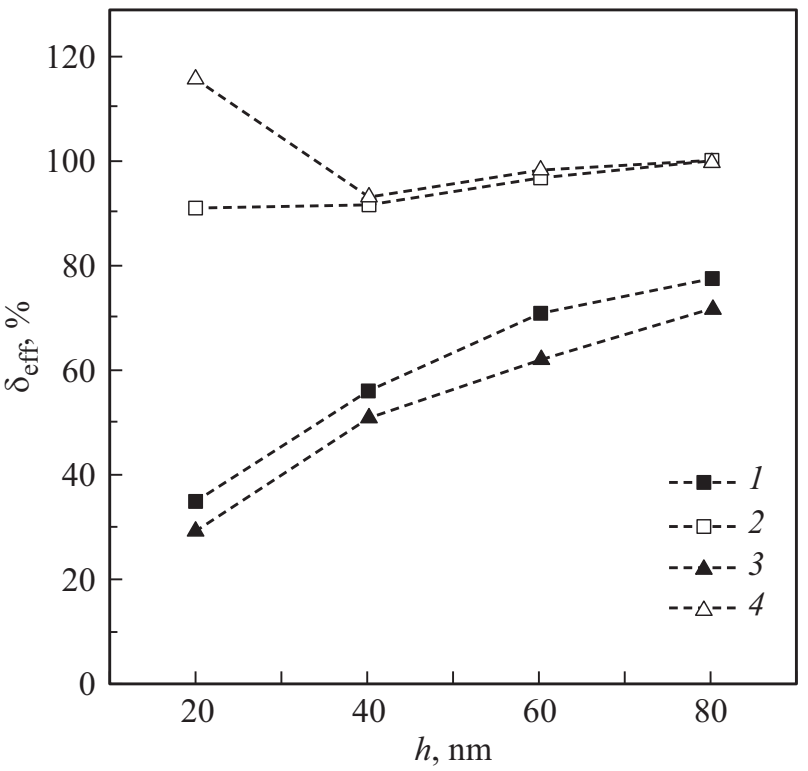

Рис. 4. Относительная доля кристаллической фазы $\delta_{\text {eff }}$ для пленок $\mathrm{Pt}$, исходной $(1,3)$ и подвергнутой отжигу $500^{\circ} \mathrm{C} / 60 \mathrm{~min}(2,4)$ в зависимости от толщины пленки $h$. Значение $\delta_{\text {eff }}$ для $80 \mathrm{~nm}$ пленки, подвергнутой отжигу, принято

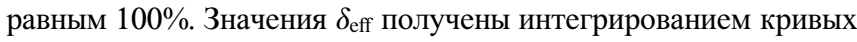
качания $(1,2)$ и путем вычисления интегральной интенсивности пика Pt (111) на $\theta-2 \theta$ дифрактограмме $(3,4)$.

худшим качеством текстуры по сравнению с пленками, подвергнутыми отжигу. Таким образом, показано преимущество метода вычисления $\delta_{\mathrm{eff}}(h)$, основанного на анализе кривых качания, позволяющее учитывать различие качества текстуры разных образцов.

Зависимости $\delta_{\text {eff }}(h)$, вычисленные с помощью кривых качания, позволяют сделать два утверждения. Во- 
первых, для исходной пленки $\delta_{\text {eff }}$ сублинейно увеличивается с ростом $h$. Во-вторых, для всех толщин $\delta_{\text {eff }}$ в результате отжига увеличивается, причем, увеличение это тем больше, чем меньше $h$. Первый факт можно объяснить уменьшением площади межзеренных границ, обусловленным увеличением латеральных размеров зерен с ростом $h$ [26]. По той же причине рост обычных и вторичных зерен в процессе отжига приводит для всех $h$

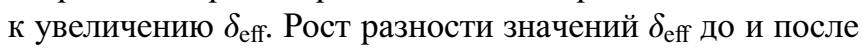
отжига с уменьшением $h$ может быть объяснен теми же причинами, что и аналогичное улучшение текстуры. Выше уже указывалось, что средний латеральный размер и суммарная занимаемая площадь вторичных зерен увеличиваются с уменьшением $h$. При $h=20 \mathrm{~nm}$ площадь, занимаемая вторичными зернами, примерно равна площади, занимаемой обычными зернами, а средний латеральный размер вторичных зерен примерно на порядок больше такового для обычных зерен. При этом средний латеральный размер обычных зерен, как и до отжига, уменьшается с уменьшением $h$. Несмотря на последний факт, можно предположить, что из-за увеличения размеров вторичных зерен при уменьшении $h$ суммарная площадь межзеренных границ уменьшится в результате отжига тем больше, чем меньше $h$, что приведет к увеличению разности значений $\delta_{\text {eff }}$ до и после отжига с уменьшением $h$.

Обращает на себя внимание факт значительного (десятки процентов) увеличения $\delta_{\text {eff }}$ с ростом $h$ или в результате отжига. Существенные изменения $\delta$ при небольших изменениях размеров зерен являются, однако, типичными для нанокристаллических материалов $[14,15,27]$.

\section{2. Нахождение распределений угла рассеяния текстуры и относительной доли кристаллической фазы по глубине для исходной пленки $\mathrm{Pt}$}

Ранее было установлено, что для исходных образцов $\Delta \gamma_{\text {eff }}$ и $\delta_{\text {eff }}$ меняются с изменением $h$, это является признаком неоднородности распределения данных параметров по глубине Pt. Такая особенность пленок хорошо известна, и нахождение данных распределений является актуальной задачей, решение которой необходимо, например, для изучения напряжений в пленках [25]. Имеющиеся данные позволяют провести оценку этих распределений для исходных образцов $\mathrm{Pt}$ при условии, что микроструктура, а следовательно, распределения $\Delta \gamma(t)$ и $\delta(t)$ в объеме растущей пленки не меняются в процессе ее осаждения. Изменения микростуктуры в растущих пленках происходят за счет термически активируемого роста зерен, который сопровождается движением межзеренных границ. Известно, что для пленок ГЦК металлов межзеренные границы неподвижны при гомологической температуpe ниже 0.2-0.3 [28]. В нашем эксперименте пленка $\mathrm{Pt}$ осаждалась при гомологической температуре 0.15 , следовательно, поставленное выше условие соблюдается. Неизменность микроструктуры пленки Pt при такой температуре подтверждается СЭМ изображениями пленок, полученными с интервалом в несколько месяцев: за этот период не было отмечено изменения морфологии поверхности. Отжиг пленок Pt происходил при гомологической температуре 0.4 , в результате чего, как было показано выше, произошли изменения кристаллической структуры, тем более существенные, чем меньше $h$. Следовательно, для пленок, подвергнутых отжигу, принципиально невозможно определить $\Delta \gamma(t)$ и $\delta(t)$.

Так как исследуемые образцы были изготовлены в одинаковых условиях, мы будем считать, что функция $D(t, \gamma)$ для всех исходных образцов одинакова. Это дает возможность рассмотреть пленку толщиной $h$ как состоящую из $N$ горизонтальных слоев равной толщины $\Delta h$, в нашем случае $\Delta h=20 \mathrm{~nm}$. Значения $\Delta \gamma$ и $\delta$ в слое между $t-\Delta h$ и $t$ можно оценить, вычтя с учетом необходимых поправок кривую качания для пленки толщиной $t-\Delta h$ из кривой качания для пленки толщиной $t$. Далее, чтобы учесть поглощение в $\mathrm{Pt}, \Delta \gamma(t)$ и $\delta(t)$ были вычислены в приближении ступенчатой функции $D(t)$ : внутри каждого слоя $D(t)$ полагалась равной константе. Таким образом, зависимости $\Delta \gamma(t)$ и $\delta(t)$ также получаются ступенчатыми, и для их нахождения предлагается следующая процедура. При сделанных выше допущениях формула (3) для кривой качания может быть записана в виде

$$
\begin{aligned}
& R C(h, \gamma)-R C_{b g}(h, \gamma)=r c(h, \gamma)=\frac{I_{0} S_{0} L}{\sin \gamma} \sum_{i=1}^{N} D\left(t_{i}, \gamma\right) \\
& \times \exp (-h / L)\left[\exp \left(t_{i} / L\right)-\exp \left(t_{i-1} / L\right)\right] .
\end{aligned}
$$

В (6) $t_{0}=0, t_{N}=h$. Например, для разбиения $60 \mathrm{~nm}$ пленки на $N=3$ слоя получаем систему

$$
\left\{\begin{aligned}
r c\left(h_{1}, \gamma\right)= & A(\theta, \gamma) D\left(t_{1}, \gamma\right) \\
r c\left(h_{2}, \gamma\right)= & A(\theta, \gamma)\left[D\left(t_{2}, \gamma\right)+D\left(t_{1}, \gamma\right) \exp (-\Delta h / L)\right] \\
r c\left(h_{3}, \gamma\right)= & A(\theta, \gamma)\left[D\left(t_{3}, \gamma\right)+D\left(t_{2}, \gamma\right) \exp (-\Delta h / L)\right. \\
& \left.+D\left(t_{1}, \gamma\right) \exp (-2 \Delta h / L)\right],
\end{aligned}\right.
$$

где $A(\theta, \gamma)=I_{0} S L[1-\exp (-\Delta h / L)] / \sin \gamma$. Решение системы (7) имеет вид

$$
\left\{\begin{array}{l}
D\left(t_{1}, \gamma\right)=r c\left(h_{1}, \gamma\right) / A(\theta, \gamma), \\
D\left(t_{2}, \gamma\right)=\left[r c\left(h_{2}, \gamma\right)-r c\left(h_{1}, \gamma\right) \exp (-\Delta h / L)\right] / A(\theta, \gamma), \\
D\left(t_{3}, \gamma\right)=\left[r c\left(h_{3}, \gamma\right)-r c\left(h_{2}, \gamma\right) \exp (-\Delta h / L)\right] / A(\theta, \gamma)
\end{array}\right.
$$



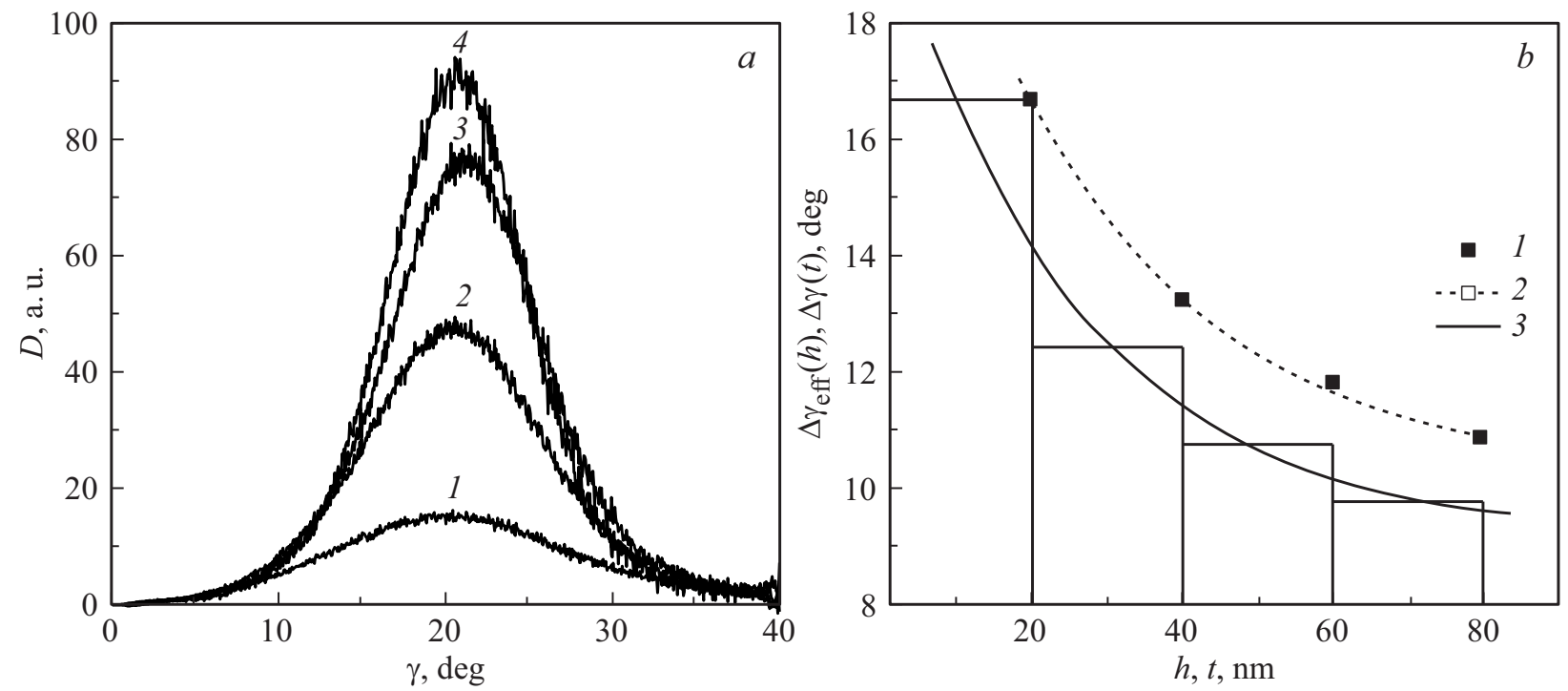

Рис. 5. $a$ - распределения ориентировок атомных плоскостей $(111) D(\gamma)$ для слоев исходной пленки: 0-20 (1), 20-40 (2), 40-60 (3), 60-80 nm (4). $b$ - зависимость $\Delta \gamma(t)$ (столбчатая диаграмма) и зависимость $\Delta \gamma_{\mathrm{eff}}(h)(1)$ для исходной пленки Рt, а также их аппроксимации функцией $\Delta \gamma(x)=[\Delta \gamma(0)-\Delta \gamma(\infty)] \exp \left(-x / x_{0}\right)+\Delta \gamma(\infty)(2,3)$.

Выражения для $\delta$ в каждом слое будут иметь вид

$$
\left\{\begin{aligned}
\delta\left(t_{1}\right)= & \int_{0}^{2 \theta} b\left(h_{1}\right) r c\left(h_{1}, \gamma\right) / A(\theta, \gamma) d \gamma, \\
\delta\left(t_{2}\right)= & \int_{0}^{2 \theta}\left[b\left(h_{2}\right) r c\left(h_{2}, \gamma\right)-b\left(h_{1}\right) r c\left(h_{1}, \gamma\right)\right. \\
& \times \exp (-\Delta h / L)] / A(\theta, \gamma) d \gamma, \\
\delta\left(t_{3}\right)= & \int_{0}^{2 \theta}\left[b\left(h_{3}\right) r c\left(h_{3}, \gamma\right)-b\left(h_{2}\right) r c\left(h_{2}, \gamma\right)\right. \\
& \times \exp (-\Delta h / L)] / A(\theta, \gamma) d \gamma .
\end{aligned}\right.
$$

Как и в случае со сравнением разных пленок, к $100 \%$ приравнивается наибольшее значение $\delta\left(t_{i}\right)$. В нашем случае можно найти распределения $\Delta \gamma(t)$ и $\delta(t)$ для диапазона $t=0-80 \mathrm{~nm}$, разбив $80 \mathrm{~nm}$ пленку на $N=4$ слоя. Результаты вычислений (8) и (9) представлены на рис. 5 и 6 соответственно. Для функции $D\left(t_{i}, \gamma\right)$ каждого слоя аналогично вычислению $\gamma_{\mathrm{eff}}$ были определены углы рассеяния текстуры $\Delta \gamma$ (рис. $5, b$ ).

Значения $\Delta y$ для всех $t$ меньше, чем соответствующие $\Delta \gamma_{\text {eff }}$, что объясняется учетом в последнем значении текстуры нижележащих слоев с большими значениями $\Delta \gamma$. Было найдено, что зависимости $\Delta \gamma_{\mathrm{eff}}(h)$ и $\Delta \gamma(t)$ хорошо аппроксимируются функцией $\Delta \gamma(x)=[\Delta \gamma(0)-\Delta \gamma(\infty)] \exp \left(-x / x_{0}\right)+\Delta \gamma(\infty)$, причем в обоих случаях $\Delta \gamma(\infty) \neq 0$. Это означает, что для использованного режима нанесения пленки угол рассеяния ее текстуры с дальнейшим ростом $h(t)$ будет стремиться к ненулевому значению, равному для $\Delta \gamma_{\text {eff }} 10.2^{\circ}$, а для $\Delta y-9.2^{\circ}$. Это можно объяснить либо влиянием шероховатости подложки, либо тем, что, несмотря на повышение качества текстуры с ростом $h$, вышележащие слои сохраняют определенную степень разориентировки, вероятно, за счет присутствия малоугловых границ в зернах.

На рис. $6, b$ представлена зависимость $\delta(t)$, согласно которой значение данного параметра увеличивается почти в три раза с увеличением $t$ с 20 до $60 \mathrm{~nm}$, после чего рост $\delta(t)$ прекращается. Как и в случае $\delta_{\mathrm{eff}}(h)$, такой рост обусловлен, главным образом, уменьшением площади межзеренных границ. Проверку правильности этого объяснения, а также предложенного метода определения $\delta$ можно сделать, используя оценку $\delta(t)$, основанную на следующей простой модели микроструктуры пленки. Предположим, что пленка толщиной $h$ состоит из столбчатых зерен, большинство из которых имеет высоту, равную толщине пленки. Такая микроструктура является типичной для металлических пленок, полученных методом магнетронного распыления. Рассмотрим в пленке между $t-\Delta h$ и $t$ горизонтальный слой толщиной $\Delta h$. Представим части зерен, попадающие в такой слой, в виде прямых многоугольных призм с высотами боковых граней, равными высоте слоя. Предположим, что каждая такая часть разделена на несколько областей когерентного рассеяния (ОКР). Таким образом, каждый слой представляет собой систему некогерентно рассеивающих блоков, высотой $\Delta h$ каждый, состоящих из нескольких ОКР, разделенных большеугловыми межзеренными границами. Такие границы будем рассматривать как прослойки аморфного материала между блоками (рис. $6, a$ ). Так как данная модель предназначена для описания зависимости $\Delta(t)$, в которой уже учтены все имеющиеся в слое ориентации зерен, можно считать, что во всех блоках атомные плоскости (111) ориентированы 

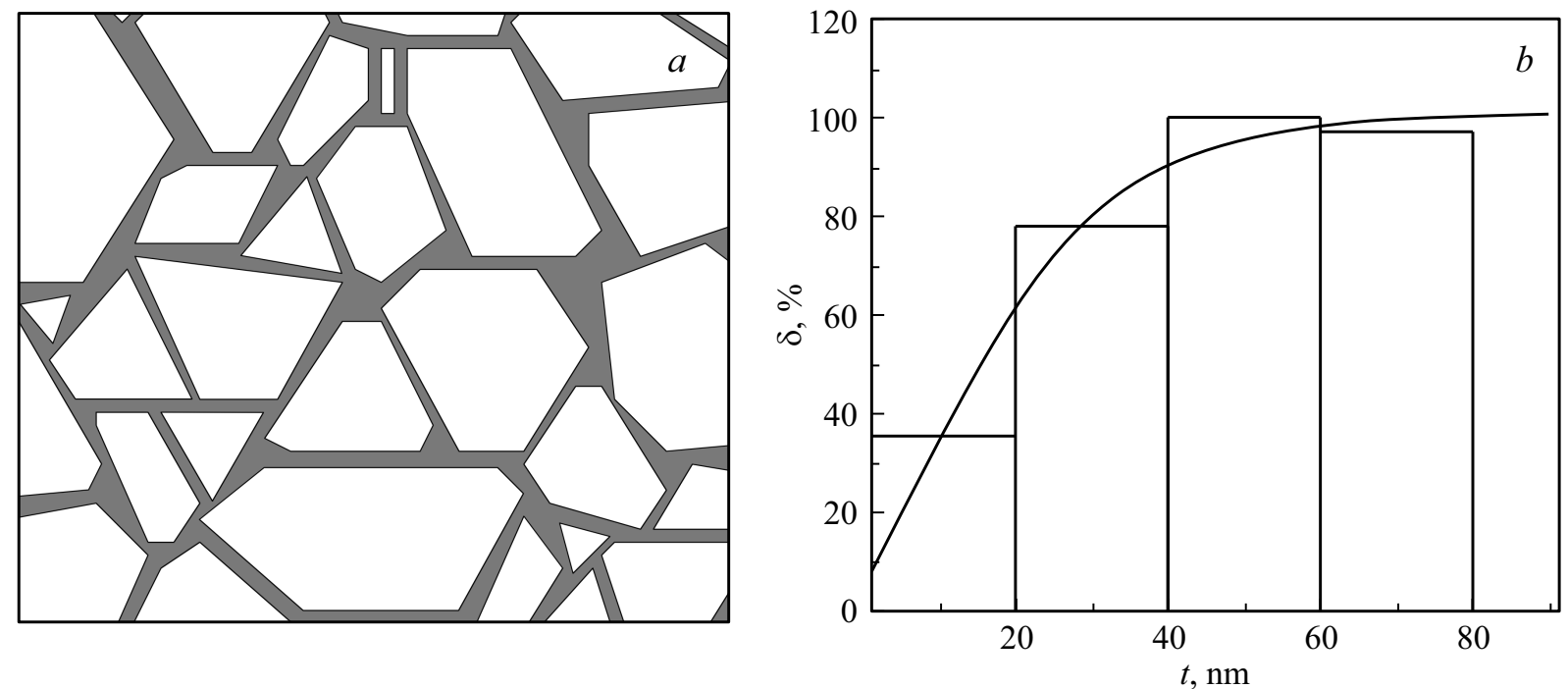

Рис. 6. $a$ - модель $20 \mathrm{~nm}$ слоя в виде системы некогерентно рассеивающих блоков в аморфной матрице (вид сверху). Белые области - зерна, серые области - аморфная прослойка между зернами. $b$ - зависимость относительной доли кристаллической фазы $\delta(t)$. Столбчатая диаграмма - экспериментальные данные, линия - аппроксимация экспериментальных данных функцией (12).

параллельно слою. Предположим, что в таком слое с площадью верхней грани $S$ содержится $P$ таких блоков со средним латеральным размером, равным $\langle l\rangle$. Выразим объем слоя $V$ как

$$
V=S \Delta h \approx P\left(\langle l\rangle+d_{g b}\right)^{2} \Delta h,
$$

где $d_{g b}$ - эффективная толщина межзеренной границы, такая, что верхние грани $P$ блоков со средним латеральным размером $\langle l\rangle+d_{g b}$ имели бы суммарную площадь, равную $S$. Объем кристаллической фазы $V_{c}$ в $V$ будет равен

$$
V_{c} \approx P\langle l\rangle^{2} \Delta h=\frac{V}{\left(1+d_{g b} /\langle l\rangle\right)^{2}} .
$$

В работе [26] были найдены значения среднего латерального размера зерна $\langle l\rangle$ для исходных пленок разных $h$, и был сделан вывод о приблизительно линейном росте $\langle l\rangle$ с ростом $h$, однако в литературе есть данные, что $\langle l\rangle$ растет как $h^{1 / n}$, где в зависимости от условий роста пленки n может принимать значения 2 [28], 3, 4 [29]. При аппроксимации экспериментальных результатов работы [26] зависимостью $h^{1 / n}$ найдено, что наилучшее соответствие данным эксперимента достигается при $n=3$, поэтому было принято, что $\langle l\rangle=k t^{1 / 3}$ (рис. 7). Необходимо указать, что наилучшее соответствие модели и эксперимента было получено в предположении, что $d_{g b}$ экспоненциально уменьшается с ростом $h$. Таким образом, выражение для $\delta(t)$ принимает вид

$$
\delta=V_{c} / V=\frac{1}{\left\{1+\frac{\left[d_{g b}(0)-d_{g b}(\infty)\right] \exp \left(-t / t_{0}\right)+d_{g b}(\infty)}{k t^{1 / 3}}\right\}^{2}} .
$$

Так как было принято, что каждый блок состоит из нескольких ОКР, следовательно, рассеивает неко-

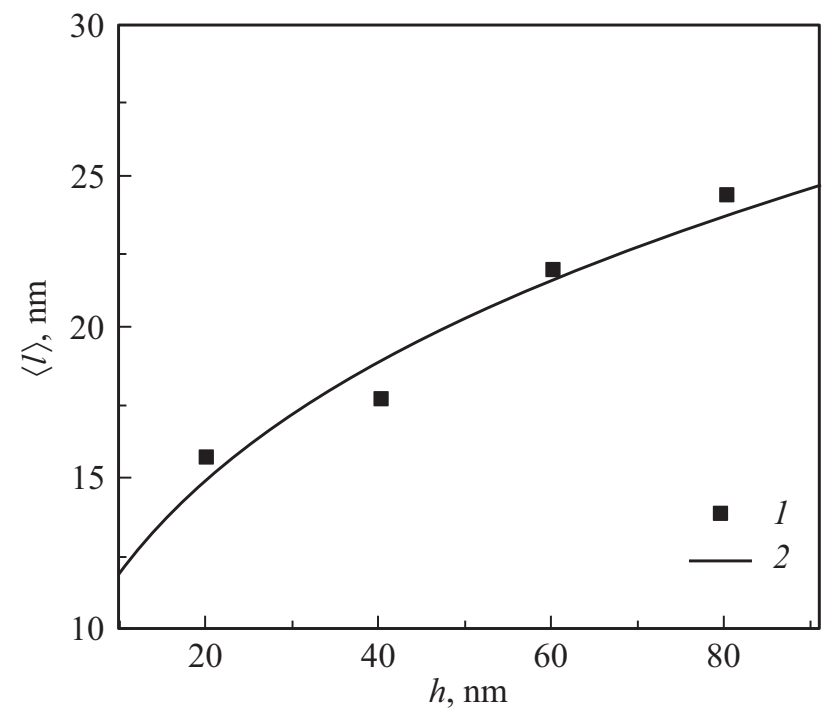

Рис. 7. Зависимость среднего латерального размера зерна $\langle l\rangle$ исходной пленки Pt от толщины $h(1)[26]$ и ее аппроксимация функцией $\langle l\rangle=k t^{1 / 3}(2)$, где $k=5.5 \mathrm{~nm}^{2 / 3}$.

герентно, интенсивность излучения, дифрагированного на каждом таком блоке, будет прямо пропорциональна объему кристаллической фазы в нем. Таким образом, интенсивность излучения, дифрагированного в слое, будет прямо пропорциональна $V_{c}$. При сделанных выше допущениях функция (12) (кривая 2 на рис. 6, b) удовлетворительно описывает экспериментальные данные. С помощью полученных в результате аппроксимации значений параметров в (12) была найдена зависимость $d_{g b}(t)$. Было получено, что эффективная ширина межзеренной границы уменьшается от 2.5 до $0.5 \mathrm{~nm}$ при 
увеличении $t$ от 40 до $80 \mathrm{~nm}$. Значение $d_{g b}$ для $t=20 \mathrm{~nm}$ равно $9 \mathrm{~nm}$, что представляется завышенным. Такое значение $d_{g b}$ может быть объяснено пористостью $20 \mathrm{~nm}$ пленки. Это подтверждается СЭМ изображениями данной пленки, на которых видны поры с латеральными размерами, примерно равными размерам зерен [26]. Для больших значений $h$ значение $d_{g b}$ согласуется с таковым для пленок и объемных нанокристаллических ГЦК металлов $[27,30]$.

Таким образом, результаты эксперимента согласуются с простой моделью микроструктуры пленки, однако значение $\delta$ для $20 \mathrm{~nm}$ пленки представляется заниженным из-за ее пористости. Последний вывод справедлив и для значения $\delta_{\text {eff }}$ при $20 \mathrm{~nm}$.

\section{Заключение}

В работе предложена и опробована методика определения относительной доли кристаллической фазы $\delta_{\text {eff }}$ в текстурированных пленках с помощью кривых качания. Для пленок Pt с толщинами $h=20,40,60,80 \mathrm{~nm}$ до и после их отжига в вакууме в режиме $500^{\circ} \mathrm{C} / 60 \mathrm{~min}$ были вычислены углы рассеяния текстуры $\Delta \gamma_{\text {eff }}$ и $\delta_{\text {eff. }}$ Было найдено, что для исходных пленок $\Delta \gamma_{\text {eff }}(h)$ экспоненциально уменьшается, а $\delta_{\text {eff }}(h)$ сублинейно увеличивается с ростом $h$. Улучшение качества текстуры с ростом $h$ объяснено переходом от неориентированного роста кристаллитов на аморфной подложке к появлению преимущественной ориентации и последующему ориентированному росту зерен. Увеличение $\delta_{\text {eff }}$ с ростом $h$ объяснено увеличением латеральных размеров зерен и обусловленным этим уменьшением объема межзеренных границ. Найдено, что отжиг приводит к уменьшению $\Delta \gamma_{\text {eff }}$ и увеличению $\delta_{\text {eff }}$ для всех $h$, причем улучшение качества текстуры и увеличение доли кристаллической фазы тем сильнее, чем меньше $h$. Эти результаты объяснены увеличением скорости роста вторичных зерен при уменьшении толщины пленки. Для исходных пленок $\mathrm{Pt}$ предложена методика нахождения распределений $\Delta \gamma$ и $\delta$ по глубине пленки. Было найдено, что $\Delta \gamma(t)$ экспоненциально уменьшается с ростом $t$, как и $\Delta \gamma_{\mathrm{eff}}(h)$. Значение $\delta(t)$ увеличивается с ростом $t$ с 20 до $60 \mathrm{~nm}$ почти в три раза, после чего рост $\delta(t)$ прекращается. Такой результат в целом согласуется с предложенной моделью микроструктуры пленки, однако при сравнении результатов эксперимента и оценок, сделанных на основе модели, был получен вывод о завышении полученного экспериментально значения $\delta$ для $t=20 \mathrm{~nm}$. Данное завышение объяснено пористостью $20 \mathrm{~nm}$ пленки.

\section{Финансирование работы}

Работа выполнена в рамках Государственного задания ФАНО России. СЭМ исследования выполнены с использованием оборудования ЦКП „Диагностика микро- и наноструктур“ при финансовой поддержке Министерства образования и науки Российской Федерации.

\section{Конфликт интересов}

Авторы заявляют, что у них нет конфликта интересов.

\section{Список литературы}

[1] Воротилов К.А., Жигалина О.М., Васильев В.А., Сигов A.C. // ФТТ. 2009. Т 51. Вып. 7. С. 1268-1271. [Vorotilov K.A., Zhigalina O.M., Vasil'ev V.A., Sigov A.S. // Phys. Solid State. 2009. Vol. 51. N 7. P. 1337-1340. Doi: 10.1134/S106378340907004X]

[2] Chateigner D., Wenk H.-R., Patel A., Todd M., Barber D.J. // Int. Ferroelectrics 1998. Vol. 19. N 1-4. P. 121-140. Doi: $10.1080 / 10584589808012699$

[3] Potrepka D.M., Fox G.R., Sanchez L.M., Polcawich R.G. // Mat. Res. Soc. Symp. Proc. 2011. Vol. 1299. P. 67-72. Doi: 10.1557/opl.2011.53

[4] Silva J.P.B., Sekhar K.C., Almeida A., Agostinho Moreira J., Martin-Sanchez J., Pereira M., Khodorov A., Gomes J.M. // J. Appl. Phys. 2012. Vol. 112. N 4. P. 044105.

Doi: $10.1063 / 1.4748288$

[5] Mirica E., Kowach G., Evans P., Du H. // Cryst. Growth \& Design. 2004. Vol. 4. N 1. P. 147-156. Doi: 10.1021/cg025595j

[6] Kim S.-K., Jeong S.-Y., Cho C.-R. // Appl. Phys. Lett. 2003. Vol. 82. N 4. P. 562-564. Doi: 10.1063/1.1536253

[7] Lin R.-C., Kao K.-S., Cheng C.-C., Chen Y.-C. // Thin Solid Films. 2008. Vol. 516. N 16. P. 5262-5265. Doi 10.1016/j.tsf.2007.07.105

[8] Caliendo C., Massimilano Latino P. // Thin Solid Films. 2011. Vol. 519. N 19. P. 6326-6329. Doi: 10.1016/j.tsf.2011.04.017

[9] Thompson C.V. // J. Appl. Phys. 1985. Vol. 58. N 2. P. 763-772. Doi: 10.1063/1.336194

[10] Keblinski P., Wolf D., Phillpot S.R., Gleiter H. // Scripta Mater. 1999. Vol. 41. N 6. P. 631-636. Doi: 10.1016/S1359-6462(99)00142-6

[11] Malek M.F., Mamat M.H., Khusaimi Z., Sahdan M.Z., Musa M.Z., Zainun A.R., Suriani A.B., Md Sin N.D., Abd Hamid S.B., Rusop M. // J. Alloys and Compounds. 2014. Vol. 582. N 5. P. 12-21. Doi: 10.1016/j.jallcom.2013.07.202

[12] Ho M.-Y., Gong H., Wilk G.D., Busch B.W., Green M.L., Voyles P.M., Muller D.A., Bude M., Lin W.H., See A., Loomans M.E., Lahiri S.K., Raisanen P.I. // J. Appl. Phys. 2003. Vol. 93. N 3. P. 1477-1481. Doi: 10.1063/1.1534381

[13] Heiroth S., Frison R., Rupp J.L.M., Lippert T., Meier E.J.B., Gubler E.M., Dobeli M., Conder K., Wokaun A., Gauckler L.J. // Solid State Ionics. 2011. Vol. 191. N 1. P. 12-23. Doi: 10.1016/j.ssi.2011.04.002

[14] Palumbo G., Thorne S.J., Aust K.T. // Scr. Metall. Mater. 1990. Vol. 24. N 7. P. 1347-1350. Doi: 10.1016/0956-716X(90)90354-J

[15] Yamasaki T. // Scripta Mater. 2001. Vol. 44. N 8-9. P. $1497-$ 1502. Doi: 10.1016/S1359- 6462(01)00720-5

[16] Roebben G., Sarbu C., Lubec T., Van der Biest O. // Mat. Sci. Eng. A. 2004. Vol. 370. N 1-2. P. 453-458. Doi: $10.1016 /$ j.msea.2003.05.004

[17] Cullity B.D. Elements of $X$-ray diffraction. Addison-Wesley Publishing Company, Inc. 1956.

[18] Уманский Я.С., Скаков Ю.А., Иванов А.Н., Расторгуев Л.Н. Кристаллография, рентгенография и электронная микроскопия. М.: Металлургия, 1982. 632 с. 
[19] Платина, ее сплавы и композиционные материалы / Под ред. Е.В. Васильевой. М.: Металлургия, 1980. 296 с.

[20] Schulz L.G. // J .Appl. Phys. 1949. Vol. 20. N 11. P. 1030-1033. Doi: 10.1063/1.1698268

[21] Физические величины. Справочник. / Под ред. И.С. Григорьева, Е.3. Мейлихова. М.: Энергоатомиздат, 1991. 1232 с.

[22] Fox A.J., Drawl B., Fox G.R., Gibbons B.J., TrolierMcKinstry S. // IEEE Trans. on UFFC. 2015. Vol. 62. N 1. P. 56-61. Doi: 10.1109/TUFFC.2014.006671

[23] Martin F., Muralt P., Dubois M.-A., Pezous A. // J. Vac. Sci. Technol. A. 2004. Vol. 22. N 2. P. 361-365. Doi: $10.1116 / 1.1649343$

[24] Petrov I., Barna P.B., Hultman L., Greene. J.E. // J. Vac. Sci. Technol. A. 2003. Vol. 21. N 5. P. S117-S128. Doi: $10.1116 / 1.1601610$

[25] Kuru Y., Welzel U., Mittemeijer E.J. // Appl. Phys. Lett. 2014. Vol. 105. N 22. P. 221902. Doi: 10.1063/1.4902940

[26] Селюков Р.В., Наумов В.В., Васильев С.В. // ЖТФ. 2018. Т. 88. Вып. 6. С. 926-933.

[27] Wei Y., Su C., Anand L. // Acta Mater. 2006. Vol. 54. N 12. P. 3177-3190. Doi: 10.1016/j.actamat.2006.03.007

[28] Thompson C.V. // Annu. Rev. Mater. Sci. 2000. Vol. 30. P. 159-190. Doi: 10.1146/annurev.matsci.30.1.159

[29] Ruffino F., Grimaldi M.G., Bongiorno C., Giannazzo F., Roccaforte F., Raineri V., Spinella C. // J. Appl. Phys. 2009. Vol. 105. N 5. P. 054311. Doi: 10.1063/1.3093681

[30] Shen T.D., Koch C.C., Tsui T.Y., Pharr G.M. // J. Mater. Res. 1995. Vol. 10. N 11. P. 2892-2896.

Doi: 10.1557/JMR.1995.2892 